\title{
Orientações de enfermagem junto à criança em tratamento quimioterápico antineoplásico
}

\author{
Nursing orientation given to children in antineoplastic chemotherapy treatment
}

Orientaciones de enfermería al niño en tratamiento quimioterápico antineoplásico

Elaine Freire Cruz ${ }^{1}$, Liliane Faria da Silva ${ }^{2}$, Fernanda Garcia Bezerra Goes ${ }^{3}$, Rosane Cordeiro Burla Aguiar ${ }^{4}$, Juliana Rezende Montenegro Medeiros de Moraes ${ }^{5}$

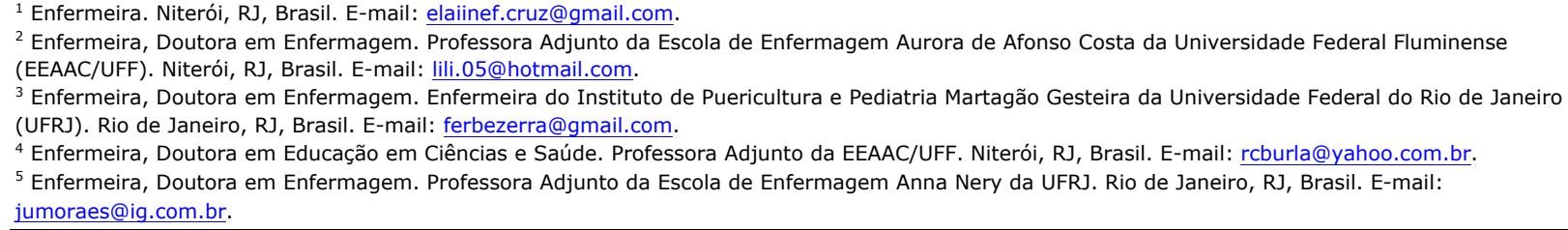

${ }^{2}$ Enfermeira, Doutora em Enfermagem. Professora Adjunto da Escola de Enfermagem Aurora de Afonso Costa da Universidade Federal Fluminense (EEAAC/UFF). Niterói, RJ, Brasil. E-mail: lili.05@hotmail.com.

${ }^{3}$ Enfermeira, Doutora em Enfermagem. Enfermeira do Instituto de Puericultura e Pediatria Martagão Gesteira da Universidade Federal do Rio de Janeiro (UFRJ). Rio de Janeiro, RJ, Brasil. E-mail: ferbezerra@gmail.com.

${ }^{4}$ Enfermeira, Doutora em Educação em Ciências e Saúde. Professora Adjunto da EEAAC/UFF. Niterói, RJ, Brasil. E-mail: rcburla@yahoo.com.br.

${ }^{5}$ Enfermeira, Doutora em Enfermagem. Professora Adjunto da Escola de Enfermagem Anna Nery da UFRJ. Rio de Janeiro, RJ, Brasil. E-mail: jumoraes@ig.com.br.

\section{RESUMO}

Pesquisa qualitativa descritiva, desenvolvida em um hospital público pediátrico, a partir de entrevista semiestruturada realizada com nove profissionais de enfermagem. Os objetivos foram identificar as orientações feitas pela enfermagem junto às crianças em idade escolar sobre a quimioterapia antineoplásica e discutir as dificuldades e facilidades na realização dessas orientações. Os resultados apontaram que as crianças recebem poucas informações sobre os efeitos colaterais do tratamento, pois as demais orientações são direcionadas aos seus pais. A relação da criança com seus pares e familiares é facilitadora da orientação e tal relação é mediadora da melhor aceitação do tratamento por parte da criança. Os profissionais apontaram como dificuldade o fato de a criança não entender a linguagem utilizada por eles durante a orientação. Conclui-se que os profissionais reconhecem que as crianças são capazes de compreender as orientações, porém encontram dificuldades em se comunicar em linguagem acessível de acordo com o desenvolvimento infantil.

Descritores: Enfermagem Pediátrica; Quimioterapia; Neoplasias; Comunicação em Saúde.

\section{ABSTRACT}

A qualitative descriptive study carried out in a public pediatric hospital using semi-structured interviews with nine nursing professionals. It aims to identify orientation given by the nursing staff to school-aged children regarding antineoplastic chemotherapy and the aspects which difficult and facilitate such orientation. The results point to the fact that children receive little information about the side effects of their treatment, for all other information is directed to parents. The child's relationship with their peers and family members facilitates orientation and such relationship serves as a mediator for better acceptance of treatment on the child's behalf. The fact that the children do not understand the language used by nursing professionals during orientation was pointed out as a hindering factor. In conclusion, professionals recognize that children are capable of understanding the given orientation, but find it difficult to communicate with them using accessible language, in accordance with the child's development.

Descriptors: Pediatric Nursing; Drug Therapy; Neoplasms; Health Communication.

\section{RESUMEN}

Investigación cualitativa, descriptiva, desarrollada en hospital público pediátrico mediante entrevistas semiestructuradas aplicas a nueve profesionales de enfermería. Se objetivó identificar las orientaciones efectuadas por la enfermería a los niños en edad escolar sobre la quimioterapia antineoplásica y discutir las dificultades y facilidades de realización de tales orientaciones. Los resultados expresaron que los niños reciben escasas informaciones sobre efectos colaterales del tratamiento, siendo tales informaciones dirigidas a sus padres. La relación del niño con pares y familiares facilita la orientación, y dicha relación es mediadora de una mejor aceptación del tratamiento por parte del niño. Los profesionales determinaron como dificultad el hecho de que el niño puede no entender el lenguaje utilizado por ellos durante la orientación. Se concluye en que los profesionales reconocen que los niños son capaces de comprender las orientaciones, aunque encuentran dificultades en comunicarse en un lenguaje accesible, de acuerdo al desarrollo infantil.

Descriptores: Enfermería Pediátrica; Quimioterapia; Neoplasias; Comunicación en Salud. 


\section{INTRODUÇÃo}

O câncer infantil corresponde a um grupo de várias doenças que têm em comum a proliferação descontrolada de células anormais. No Brasil, é a segunda causa de morte em crianças e adolescentes, entre um e dezenove anos de idade, estando a primeira relacionada a acidentes e violência(1). Além disso, é um acontecimento devastador que provoca mudanças e reações inesperadas, não apenas na vida da criança, mas também na vida de seus familiares ${ }^{(2)}$.

Em nosso pais, estima-se que $70 \%$ das crianças com câncer podem ser curadas se diagnosticadas precocemente e tratadas adequadamente, em centros especializados, tendo a maioria uma boa qualidade de vida após o tratamento(1). Já nos Estados Unidos, estimou-se que cerca de 11.630 crianças com menos de quinze anos de idade seriam diagnosticadas com câncer em 2013. Com o tratamento adequado, cerca de $80 \%$ dessas crianças poderão sobreviver por cinco anos ou mais $^{(3)}$.

A assistência em pediatria oncológica baseia-se no cuidado preventivo, curativo e paliativo. O cuidado preventivo pode se dar por ações antes do nascimento da criança, através do aconselhamento genético, e durante a infância, a partir de orientações de hábitos de vida saudáveis: alimentação, atividade física e cuidados com o meio ambiente. Entretanto, vale ressaltar que a associação entre câncer infantil e fatores de risco ainda não está bem esclarecida. O cuidado curativo engloba o diagnóstico, o tratamento e o controle; já o paliativo é adotado quando a possibilidade de cura é afastada ${ }^{(4)}$.

O tratamento com objetivo curativo é longo e demanda cuidados e mudança no cotidiano de vida, por isso, quanto mais informada a criança for acerca da doença e dos cuidados que precisa ter, respeitando seu grau de desenvolvimento, mais facilitado será seu tratamento $^{(5)}$.

Existem várias modalidades de tratamento para o câncer infantil, sendo as principais a quimioterapia, a cirurgia e a radioterapia, aplicadas dependendo do tipo de tumor e de acordo com a extensão da doença ${ }^{(1)}$. No entanto, a quimioterapia é a modalidade mais utilizada, podendo ser associada ou não às outras modalidades de tratamento $^{(5)}$.

Atualmente, parte do tratamento do câncer é realizado em nível ambulatorial, favorecendo a permanência da criança no convívio familiar ${ }^{(6)}$. Nesse sentido, os efeitos colaterais da quimioterapia, tais como fadiga, anorexia, náuseas, vômito, diarreia, dor, mucosite, disúria, perda de peso e alopecia ${ }^{(7)}$, surgem em casa e são fontes geradoras de estresse para a criança e sua família( ${ }^{(8)}$.

Destaca-se que o tratamento da criança com câncer deve ser abrangente, exigindo atenção a suas necessidades físicas, psicológicas e sociais, além da inclusão da família e da busca pela promoção de cuidados atraumáticos e garantia do direito à informação ${ }^{(6-9)}$.

Com relação ao direito à informação, observa-se na literatura que pesquisas apontam a importância do desenvolvimento de ações educativas voltadas aos familiares da criança em quimioterapia ${ }^{(6-10)}$. Entretanto, no Brasil, pouco se discute acerca do desenvolvimento de orientações junto às crianças ${ }^{(5)}$.

Uma revisão integrativa destacou os aspectos gerenciais do enfermeiro no cuidado à criança com câncer, assim como os desafios do enfermeiro na atenção paliativa oncológica pediátrica. No estudo não houve discussão acerca do envolvimento da criança nas orientações de enfermagem quanto à quimioterapia ${ }^{(11)}$.

No âmbito internacional, um estudo realizado em três principais centros de tratamento oncológico no Reino Unido, mostrou que as crianças se preocupam com seus sintomas e se incomodam com o fato de a comunicação a respeito do seu adoecimento e tratamento acontecer entre os seus pais e os profissionais de saúde, sem incluí-las ${ }^{(10)}$.

Durante o tratamento oncológico, a necessidade que a criança tem com relação à comunicação e informação depende de sua idade. As crianças com menos de cinco anos, contam com os pais para comunicar-se com profissionais de saúde, já as crianças mais velhas, como os escolares entre seis e doze anos, querem se comunicar diretamente com os profissionais de saúde ${ }^{(10)}$.

Neste sentido, esta pesquisa deu enfoque às orientações feitas pela enfermagem junto às crianças em idade escolar. Esta faixa etária foi eleita, pois nesta idade elas já têm capacidade de classificar, selecionar, ordenar e organizar os fatos sobre o mundo para solucionar problemas. Desenvolvem a capacidade de ler, que é um dos o instrumentos mais importantes para a autonomia e autocuidado(12). Além disso, demostram interesse em estabelecer comunicação direta com os profissionais de saúde ${ }^{(10)}$.

Diante de tais características particulares dessa fase de desenvolvimento infantil, constata-se que os escolares já são capazes de entender sobre sua doença, seu tratamento e, assim, podem cuidar de si juntamente com seus pais ou responsáveis. Neste sentido, a equipe de enfermagem deve incluí-los, juntamente com seus 
familiares, no planejamento das suas ações educativas, tais como as orientações, como uma forma de melhorar a qualidade de vida dessas crianças.

$\mathrm{Na}$ busca de melhor compreensão do desenvolvimento infantil, esta pesquisa apoiou-se nas concepções de Vygotsky como referencial teórico ${ }^{(13)}$. Para este autor, desenvolvimento infantil consiste na capacidade de a criança transitar de um nível elementar para um nível mais elaborado de realização de tarefas e de compreender o que acontece ao seu redor. Essa transformação é processual e se dá através da mediação, com uso de signos e instrumentos, e da interação social ${ }^{(13-14)}$.

Os usos dos instrumentos e dos signos, embora diferentes, estão mutuamente ligados ao longo da evolução da espécie humana e do desenvolvimento de cada indivíduo. Quanto à diferença, é preciso esclarecer que o instrumento auxilia o homem em ações concretas. Por exemplo, a caneta é um instrumento concreto que auxilia na escrita, e o signo na atividade psicológica, como os lembretes de papel auxiliam o homem na capacidade mental de lembrar compromissos. Quanto à semelhança, ambos têm função mediadora ${ }^{(13)}$.

Esses elementos mediadores estão presentes no mundo cultural humano, sendo social e historicamente construídos pelo homem e transmitidos pelas interações sociais às crianças integrantes do grupo cultural.

No curso do desenvolvimento infantil, a criança gradativamente vai internalizando suas experiências com o mundo e as pessoas, e com isso ocorre uma transformação no seu comportamento ${ }^{(13)}$.

$\mathrm{Na}$ teoria de desenvolvimento infantil elaborada por Vygotsky, ele conceituou as zonas de desenvolvimento humano segundo a seguinte classificação: zona de desenvolvimento real, que está relacionada ao que já foi aprendido, ou seja, aquilo que a criança é capaz de fazer sozinha; zona de desenvolvimento potencial, aquela que está relacionada com aquilo que pode ser aprendido com a ajuda do outro, seja um adulto ou uma criança mais experiente; e a zona de desenvolvimento proximal, o intervalo entre as duas zonas de desenvolvimento já citadas $^{(13)}$.

O papel do adulto na promoção do desenvolvimento infantil é o de agir em uma relação de mediação, criando condições para que as crianças se desenvolvam. Dessa forma, cabe ao profissional de saúde interagir socialmente com a criança em tratamento quimioterápico numa relação de mediação para favorecer seu aprendizado e compreensão acerca do seu tratamento.
Com base no exposto, a presente pesquisa teve como objeto de estudo orientações de enfermagem às crianças em idade escolar em tratamento quimioterápico antineoplásico. Os objetivos foram os seguintes: (a) identificar as orientações feitas pela enfermagem junto às crianças em idade escolar sobre a quimioterapia antineoplásica e (b) discutir as dificuldades e facilidades que a enfermagem encontra na realização de orientações à criança em idade escolar sobre o tratamento de quimioterapia antineoplásica.

\section{MÉTODO}

Trata-se de uma pesquisa qualitativa descritiva ${ }^{(15)}$. 0 cenário foi o setor de internação de um hospital público pediátrico localizado no Estado do Rio de Janeiro, que atende crianças em tratamento oncológico durante ciclos de quimioterapia.

Os sujeitos foram nove profissionais da equipe de enfermagem, sendo três enfermeiros e seis técnicos de enfermagem que realizam suas atividades no setor eleito para a pesquisa. Trabalhamos com as duas categorias profissionais por estarem presentes durante toda a internação da criança, participando dos cuidados de enfermagem realizados tanto na administração de quimioterapia antineoplásica, como nas orientações para o tratamento.

Delimitaram-se, como critérios de inclusão, profissionais da equipe de enfermagem que atuavam na assistência à criança em quimioterapia por mais de seis meses, pois assim teriam tempo de convivência com estas crianças e poderiam falar de suas experiências, facilidades e dificuldades. Foram excluídos da pesquisa os profissionais da equipe de enfermagem em férias, licença médica ou qualquer outro tipo de afastamento do setor.

O número de participantes foi definido no decorrer do trabalho de campo onde, por meio da organização dos depoimentos, buscou-se a identificação da saturação dos dados, ou seja, a existência de redundância e repetição de ideias, padrões de comportamento, e visões de mundo ${ }^{(16)}$.

A coleta de dados foi realizada em dezembro de 2012, por meio de uma entrevista semiestruturada. Foi utilizado um roteiro de entrevista, em que constavam perguntas abertas e fechadas. As perguntas fechadas foram cruciais para a identificação dos sujeitos. As perguntas abertas versavam sobre as orientações feitas pela equipe de enfermagem às crianças em idade escolar sobre a quimioterapia antineoplásica, suas facilidades e dificuldades para realizar as orientações. 
Tendo como base a literatura científica sobre a temática, tínhamos a priori a hipótese de que a equipe de enfermagem realizava algum tipo de orientação junto à criança em tratamento quimioterápico antineoplásico. Para o processo de análise partimos de categorias préestabelecidas e os dados obtidos foram organizados da melhor maneira possível dentro dessas categorias ${ }^{(17)}$.

Para um registro integral e preciso das falas dos sujeitos, as entrevistas foram gravadas com o auxílio de um aparelho mp3, com autorização prévia dos sujeitos. O anonimato dos participantes foi mantido durante todo o tempo; para isso, utilizamos codinomes fictícios para identificá-los. Foram usados nomes de personagens da Disney por sugestão da primeira pesquisadora com aceite dos participantes.

A pesquisa foi aprovada pelo Comitê de Ética e Pesquisa da instituição onde foi realizada (CAAE: 06776712.1.0000.5264/ Parecer: 128.935), e respeitou todos os aspectos contidos na Resolução 466/12 do Conselho Nacional de Saúde.

Neste sentido, após autorização do Comitê de Ética, foi realizado contato com os profissionais que trabalham no setor. Inicialmente a pesquisadora apresentou-se e falou sobre a pesquisa destacando seus objetivos, assim como os aspectos contidos no Termo de Consentimento Livre e Esclarecido - TCLE. Posteriormente, foi feito convite para atuarem como participantes voluntários da pesquisa. O aceite foi formalizado com a assinatura do TCLE.

Após a coleta de dados, as falas dos entrevistados foram transcritas na íntegra e os dados analisados por meio da análise de conteúdo, que pode ser definida como uma técnica de investigação das comunicações que utiliza procedimentos objetivos e sistemáticos de descrição do conteúdo das mensagens com a finalidade de interpretar essas mesmas comunicações ${ }^{(17)}$.

A analise dos dados foi realizada seguindo as três fases da análise de conteúdo: (a) pré-análise, quando realizamos a leitura flutuante para conhecer o conteúdo do material empírico gerado pelas entrevistas deixandonos invadir por impressões e orientações; (b) fase de exploração do material, quando a partir de leituras exaustivas os dados brutos foram transformados em unidades que representavam significados e depois agregados nas categorias pré-estabelecidas que responderam aos objetivos e hipótese da pesquisa; (c) fase de tratamento e a interpretação dos resultados, quando foi possível fazer inferências à luz da literatura científica sobre orientações a crianças em tratamento quimioterápico ${ }^{(17)}$.

\section{RESULTADOS E DISCUSSÃO}

Da análise de conteúdo resultaram as seguintes categorias: orientações feitas pela enfermagem à criança em idade escolar e seus familiares sobre a quimioterapia; facilidades para realização das orientações à criança em idade escolar sobre a quimioterapia; e dificuldades para realização das orientações sobre quimioterapia à criança em idade escolar.

\section{Orientações feitas pela enfermagem à criança em idade escolar e seus familiares sobre a quimioterapia}

A partir das respostas dos sujeitos em relação às orientações feitas pela enfermagem sobre a quimioterapia, percebemos que a maior parte dos profissionais realizam as orientações direcionando-as para os pais e poucos profissionais orientam as crianças em idade escolar sobre a quimioterapia a qual é submetida.

Ao falarem a respeito das orientações sobre a quimioterapia, voltadas à criança em idade escolar, houve destaque para o volume de informações:

Bom, a orientação que a gente tenta fazer para criança em relação à quimioterapia não é mentirosa, mas a gente tenta passar o mínimo possível, entendeu? (Cinderela- técnica de enfermagem)

Damos pouca orientação a respeito da importância da quimioterapia e da manutenção do acesso venoso integro e pérvio. (Bela- enfermeira)

Os profissionais têm a preocupação para que o conteúdo da informação seja verdadeiro e evitam mentiras, entretanto afirmam que volume de informação é pouca, o mínimo possível. Sendo assim, na interação social com as crianças, a atuação do profissional na zona de desenvolvimento potencial infantil(13), ou seja, naquela que precisa da ajuda de alguém mais experiente, é reduzida com relação ao volume de informações.

Embora a maioria dos profissionais entrevistados realize orientações junto aos pais, destaca-se aqui que, já no primeiro contato com a criança, ela deve ser incluída na conversa e no cuidado, a partir de indagações a respeito do seu nome, idade ou outras informações, para que se sinta segura e possa expressar o que sente $^{(12)}$. Além disso, as crianças são capazes de compreender e perceber, de maneira precoce, qualquer 
indício que não seja reconhecido como normal por elas próprias $^{(18)}$.

Outro aspecto a ser levado em consideração é que as crianças percebem o quanto a fase de tratamento é importante para o alcance da cura, logo, têm preocupações relacionadas à recuperação da sua saúde, assim como ao seu prognóstico. Sua compreensão acerca do adoecimento e tratamento as tornam mais colaboradoras e participantes na tomada de decisões ${ }^{(19)}$.

Dois profissionais apontaram que as crianças, nessa faixa etária, têm a capacidade de entender a necessidade do tratamento e algumas modificações que acontecem no próprio corpo. Eles falam com elas a respeito dos efeitos colaterais tais como alopecia e enjoo, no momento da administração de medicamentos:

A gente sabe que a criança nessa idade já consegue entender alguma coisa. Então a gente fala: a gente vai dar um remedinho e você vai ficar com o cabelo assim (alopecia) (Cinderela - técnica de enfermagem).

Quando vou administrar uma quimioterapia digo que aquilo é um remédio para o tratamento, que ela pode ficar enjoada (...) falo sobre o risco de queda de cabelo (Aurora - enfermeira).

$\mathrm{Na}$ interação social ${ }^{(13)}$ da criança em idade escolar com o meio em que vive, especialmente no universo do tratamento quimioterápico, ela internaliza alguns sinais e sintomas, tais como alopecia e enjoo, e é capaz de associá-los aos medicamentos que utiliza.

Com relação à fase de desenvolvimento infantil, destacamos que as crianças em idade escolar tornam-se capazes de lidar, simultaneamente, com muitos aspectos diferentes de uma situação. Elas resolvem os problemas de modo concreto, sistemático, com base em sua recepção(12). Nesta idade, a criança já entende da doença em si, tem preocupação com autoimagem e sensibilidade para sentir seu corpo como estranho ${ }^{(18)}$.

Essas características de desenvolvimento devem ser valorizadas e respeitadas pela da equipe de saúde, de forma a considerar as preferências pessoais. As crianças possuem capacidade de compreensão de sua doença e tratamento, por isso não se deve negligenciá-las tornando-as sujeitos passivos no tratamento do câncer $^{(19)}$.

Com o objetivo de minimizar os prejuízos decorrentes da percepção da autoimagem, a enfermagem pode informar à criança e à família a respeito dos efeitos que a doença e o tratamento medicamentoso podem levar para a sua vida, principalmente os efeitos visíveis aos seus olhos e aos olhos de outras pessoas, como por exemplo as mudanças corporais. É também importante encorajar a criança a compartilhar seus medos, angústias e insatisfações em relação a sua imagem com a pessoa responsável por seu cuidado, seja familiar cuidador ou profissional ${ }^{(20)}$.

Apesar das perguntas da pesquisa terem sido voltadas para as orientações realizadas junto às crianças, a maior parte dos profissionais situou em suas respostas a família e os pais, em detrimento da criança:

Orientamos a família antes mesmo de administrar a quimioterapia, (...) informamos qual é o antineoplásico que a gente vai administrar, como vai ser administrado, a velocidade de infusão, se vai ser na bomba infusora ou não, (...) a gente acaba não orientando muito a criança (Bela - enfermeira).

Eu falo para os pais que o cabelinho da criança vai cair, que a quimioterapia provoca enjoo, sobre a importância da higienização das mãos. (Jasmine - técnica de enfermagem)

Durante a quimioterapia, a gente fala para os pais sobre o que pode acontecer: a criança pode nausear, pode ter efeitos colaterais da quimioterapia, entendeu? Após a quimioterapia, há risco de queimadura, por isso orientamos os pais que a criança não pode tomar sol (Mulan- enfermeira).

O conteúdo informativo das orientações realizadas junto aos familiares e pais é diversificado, os profissionais falam qual o quimioterápico antineoplásico que será administrado, a técnica que será usada para administração, as possíveis reações dos quimioterápicos, os cuidados para evitar infecção, como a lavagem das mãos, e ainda os cuidados que precisam ter após a quimioterapia.

Em relação às orientações acerca do tratamento quimioterápico, os profissionais demonstraram que os familiares são os principais cuidadores da criança e receptores das informações transmitidas ${ }^{(6)}$. Entretanto, as crianças em idade escolar já conseguem entender a respeito de sua patologia e seu tratamento, conhecem a si mesmas e possuem a astúcia de ver quando algo está diferente ${ }^{(18)}$.

Vale destacar que a criança em idade pré-escolar não associa seus sintomas aos medicamentos utilizados na quimioterapia e sim à propria doença. A partir de seis anos de idade, porém, ela começa reconhecer que alguns sintomas que sente durante o adoecimento pelo câncer 
são decorrentes do uso dos medicamentos. Por exemplo, elas percebem que as alterações nos níveis de fome são devido ao uso esteroides. Além disso, elas falam abertamente sobre como o tratamento interfere em suas vidas, que gostariam de receber informações diretamente dos profissionais, com palavras de fácil compreensão(10).

Dessa forma, é importante que os profissionais da saúde e cuidadores conversem com elas, as orientem, levem em consideração suas queixas e utilizem-nas como norteadoras do cuidado no hospital e no domicílio, estimulando também o autocuidado ${ }^{(18)}$. Assim, poderão estabelecer uma relação de mediação na estimulação do desenvolvimento infantil ${ }^{(13)}$ voltado para as necessidades da criança em tratamento quimioterápico.

\section{Facilidades para realização das orientações à criança em idade escolar sobre a quimioterapia}

Os profissionais apontaram que o contato da criança com outras crianças e também com seus familiares é um aspecto que facilita a realização das orientações de enfermagem acerca da quimioterapia, pois a criança aceita melhor sua condição:

O contato com as outras crianças facilita, porque ela sabe que o cabelo vai cair, porque vê as outras crianças, (...) ela aceita melhor (Cinderela - técnica de enfermagem).

A família também faz o apoio psicológico. A mãe fala: "filha, você vai ficar assim, mas olha você vai ficar uma princesa. Ninguém vai deixar de te amar por causa disso. É para o seu bem, é para você ficar bem". Então, dessa forma, a criança acaba aceitando (Cinderela - técnica de enfermagem).

A forma de as crianças compreenderem o meio social em que estão inseridas é resultante de um processo de recordação de eventos que já ocorreram, ou seja, de internalizações e também de representações presentes e futuras. Elas são capazes de identificar sua doença e o que a cerca ${ }^{(13,18)}$. Neste sentido, ao se relacionarem com outras crianças no mesmo processo de adoecimento e tratamento, as crianças formam conceitos e representação, e identificam sua própria condição como semelhante a das outras crianças ${ }^{(13)}$.

A interação social da criança com os familiares é fundamental durante o tratamento do câncer, pois a família é o sistema mais importante que a criança pertence e as relações familiares são cruciais para o adequado enfrentamento da doença e do tratamento ${ }^{(6)}$.

\section{Dificuldades para realização das orientações sobre} a quimioterapia à criança em idade escolar

Os profissionais apontaram como dificuldade para realização das orientações o fato de a criança não entender a linguagem, utilizada pelo profissional durante a orientação junto ao acompanhante:

A gente acaba orientando o acompanhante. Muitas vezes a criança está do lado, mas ela não entende aquela linguagem. Eu me preocupo muito com o acompanhante e nem sempre eu chego e converso com a criança (Bela enfermeira).

Nota-se que mesmo as crianças estando ao lado do familiar nas orientações, o profissional pode não incluílas, por achar que elas não entendem a linguagem utilizada.

As orientações realizadas pelo enfermeiro para as crianças e para seus pais necessitam ter uma linguagem acessível, precisam ser de fácil compreensão, abrangendo a totalidade desses indivíduos, para que a orientação de fato provoque mudanças no cotidiano das crianças com o objetivo de melhorar sua qualidade de vida $^{(20)}$.

Um estudo buscou compreender o processo do diagnóstico à sobrevivência do câncer a partir da perspectiva da criança. Os pesquisadores identificaram que crianças em idade escolar usam termos técnicos para designar sua doença, além disso, compreendem mudanças no corpo que estão relacionados ao câncer e são capazes de falar sobre os procedimentos para diagnóstico e tratamento(19). Elas gradativamente internalizam termos técnicos e se apropriam da linguagem utilizada pelos profissionais ${ }^{(13)}$ no universo do tratamento oncológico.

Com relação à criança, o profissional precisa utilizar linguagem adequada e interagir socialmente com ela buscando auxiliar o seu processo de desenvolvimento. Contudo, esse processo não é passivo e rápido, a criança precisa de um tempo para ressignificar as informações recebidas $^{(13)}$.

O profissional, ao interagir com a criança, atua em sua zona de desevolvimento potencial como mediador da aquisição de novos conhecimentos. Para que a informação torne-se concreta, as palavras precisam fazer sentido, só assim passarão a fazer parte da zona de desenvolvimento real da criança ${ }^{(13)}$.

Para esse fim, o lúdico é reconhecido como uma medida terapêutica, que promove a continuidade do desenvolvimento infantil e possibilita o restabelecimento 
físico e emocional por tornar o processo de adoecimento menos traumatizante ${ }^{(13-14,21)}$.

Com a inclusão do lúdico no atendimento e orientação junto às crianças, o profissional deixa de focalizar apenas a doença e passa a enxergar o indivíduo como um todo, englobando, na prestação de assistência, cuidados com os aspectos psicológicos, sociais e culturais, além dos físicos. Dessa maneira, o lúdico funciona como elo entre a criança e os profissionais de saúde, caracterizando-se como uma atividade-meio, ou seja, um recurso que tem como finalidade facilitar ou conduzir aos objetivos estabelecidos ${ }^{(22)}$.

Quando foram perguntados sobre as dificuldades dos profissionais para realizarem as orientações junto às crianças, eles permaneceram falando sobre aquelas que eles têm com seus pais:

As dificuldades são muitas, (...) as mães que não aceitam a higienização das mãos, não entendem que o que a criança come na rua pode prejudicar, elas continuam trazendo coisa da rua para a criança comer e não pode! (Jasmine - técnica de enfermagem).

Nem sempre os pais entendem que a quimioterapia tem diversas reações, acho que essa é a maior dificuldade. Tem também a dificuldade de aceitação da doença (Pocahontas - técnica de enfermagem).

Os profissionais falaram das dificuldades com os pais relacionadas aos cuidados com a lavagem das mãos, com a alimentação, reações da quimioterapia e a própria dificuldade de aceitação do diagnóstico de câncer. Esse padrão de respostas pode ser indicativo da pouca familiaridade que a equipe tem com orientações voltadas às crianças, sendo mais fácil apontar outras dificuldades.

Em relação aos fatores que interferem na aceitação da patologia e tratamento de seus filhos, é importante lembrar que com o diagnóstico a maior parte dos pais sente-se desesperada, com medo da morte, culpada e revoltada ${ }^{(23)}$. Todos esses sentimentos interferem no processo de aceitação.

Na realização do cuidado e das orientações acerca da quimioterapia direcionados aos familiares, é preciso levar em consideração as percepções que eles têm sobre a doença, com vistas a favorecer a troca de informações, orientações e esclarecimentos quanto aos cuidados. Isto requer que a enfermagem promova educação e saúde ao longo de sua assistência, pautada na certeza de que a família é uma importante fonte de apoio e bem-estar para a criança com câncer ${ }^{(24)}$.

\section{CONSIDERAÇÕES FINAIS}

Apesar de as crianças serem capazes de compreender o que está acontecendo com o seu corpo em decorrência da doença e do tratamento quimioterápico, as informações que recebem dos profissionais versam principalmente sobre os efeitos colaterais e são realizadas no momento da administração do medicamento, e não de maneira contínua de forma a favorecer a aquisição de novos conhecimentos acerca de sua condição.

A maior parte dos profissionais realiza as orientações acerca da quimioterapia antineoplásica aos pais dessas crianças, perdendo a oportunidade de atuarem como mediadores de informações sobre o tratamento quimioterápico junto às crianças.

É importante ressaltar que não cabe apenas aos familiares serem mediadores do conhecimento. $O$ profissional também pode e deve assumir esse papel, levando em consideração o desenvolvimento infantil da criança em idade escolar e sua capacidade de compreender o que está sendo falado.

O profissional, além de informar sobre os efeitos do tratamento e encorajar a comunicação, pode estimular a criança a buscar estratégias que facilitem sua adaptação e melhore sua autoimagem, como o uso de adornos, chapéus, bonés e lenços coloridos com motivos infantis.

A interação social das crianças em tratamento quimioterápico antineoplásico com seus pares e seus familiares foi destacada como facilitadora do processo de orientação, pois o contato da criança com outras crianças que estão passando pelo mesmo momento que o seu facilita a aceitação da doença e da alteração da autoimagem.

Como principal dificuldade, os profissionais apontaram que as crianças não entendem a linguagem utilizada por eles enquanto orientam os familiares. Sugere-se assim que haja planejamento de realização de orientações junto às crianças com uso de linguagem e recursos adequados ao nível de desenvolvimento infantil, tais como jogos e brinquedos.

O ganho maior dessa pesquisa foi evidenciar que os profissionais reconhecem que as crianças em idade escolar são capazes de compreender as orientações sobre sua doença e tratamento, porém encontram dificuldades em se comunicar com essas crianças em linguagem acessível que respeite o nível de desenvolvimento infantil.

O profissional de saúde, principalmente a equipe de enfermagem que atua no tratamento quimioterápico, precisa entender que as orientações junto às crianças 
precisam fazer parte dos cuidados de enfermagem, pois podem contribuir para melhora de qualidade de vida das

\section{REFERÊNCIAS}

1. Instituto Nacional de Câncer José Alencar Gomes da Silva. Estimativa 2012 : incidência de câncer no Brasil [Internet]. Rio de Janeiro: INCA; 2011 [acesso em: 30 jun 2014]. Disponível em:

http://portal.saude.sp.gov.br/resources/ses/perfil/gestor/homep age/estimativas-de-incidencia-de-cancer-

2012/estimativas_incidencia_cancer_2012.pdf.

2. Sales CA, Santos GM, Santos JA, Marcon SS. O impacto do diagnóstico do câncer infantil no ambiente familiar e o cuidado recebido. Rev. Eletr. Enf. [Internet]. 2012 [acesso em: 30 jun 2014];14(4):841-9. Disponível em:

http://dx.doi.org/10.5216/ree.v14i4.15446.

3. Akard TF, Gilmer MJ, Friedman D, Given B, HendricksFerguson VL, Hinds PS. From qualitative work to intervention development in pediatric oncology palliative care research. J Pediatr Oncol Nurs. 2013;30(3):153-60.

4. Mutti CF, Paula CC, Souto MD. Assistência à Saúde da Criança com Câncer na Produção Científica Brasileira. Rev. Brasileira de Cancerologia [Internet]. 2010 [acesso em 13 out 2013]; 56(1):71-83. Disponível em:

http://www.inca.gov.br/rbc/n_56/v01/pdf/11_revisao_de_literat ura_assistencia_saude_crianca_cancer.pdf.

5. Cicogna EC, Nascimento LC, Lima RAG. Crianças e

adolescentes com câncer: experiências com a quimioterapia. Rev Lat Am Enfermagem [Internet]. 2010 [acesso em 10 mai 2011]; 18(5):864-872. Disponível em:

http://www.scielo.br/pdf/rlae/v18n5/pt_05.pdf.

6. Gomes IP, Amador DD, Collet N. A presença de familiares na sala de quimioterapia pediátrica. Rev Bras Enferm.

2012;65(5):803-808.

7. Silva MM, Silva JA, Esteves LO, Mesquita MGR, Stipp MAC, Duarte SMC. Perfil sociodemográfico e clínico de pessoas em tratamento quimioterápico: subsídios para o gerenciamento em enfermagem. Rev. Eletr. Enf. [Internet]. 2013 [acesso em 14 out 2013]; 15(3):704-12. Disponível em:

http://dx.doi.org/10.5216/ree.v15i3.18417.

8. Arruda IB, Francisco de Paula JMS; SILVA, Rêneis PL. Efeitos adversos da quimioterapia antineoplásica em crianças: o conhecimento dos acompanhantes. Cogitare enferm. [Internet]. 2009 [acesso em 14 out 2013]; 14(3):535-9. Disponível em: http://ojs.c3sl.ufpr.br/ojs2/index.php/cogitare/article/view/1618 6/10704.

9. Artilheiro APS, Almeida FA, Chacon JMF. Use of therapeutic play in preparing preschool children for outpatient chemotherapy. Acta paul. enferm. [Internet]. 2011 [acesso em 14 out 2013]; 24(5):611-616. Disponível em: http://www.scielo.br/pdf/ape/v24n5/en_03v24n5.pdf.

10. Gibson F, Aldiss S, Horstman M, Kumpunen S, Richardson A. Children and young people's experiences of cancer care: a qualitative research study using participatory methods. International Journal of Nursing Studies. 2010; 47:1397-1407. 11. Silva TP, Leite JL, Santos NLP, Silva IR, Mendonça ACA, Santos MJC, Silva, JS. Cuidados de enfermagem à criança com câncer: uma revisão integrativa da literatura. Revista de Enfermagem da UFSM [Internet]. 2013 [acesso em 14 fev 2014]; 3(1): 68-78. Disponível: http://cascavel.ufsm.br/revistas/ojs2.2.2/index.php/reufsm/article/view/6918.

12. Hockenberry MJ, Wilson D. Wong fundamentos da enfermagem pediátrica. 8 ed. Rio de Janeiro: Elsevier, 2011. 13. Vygotsky LS. A formação social da mente: o desenvolvimento dos processos psicológicos superiores. 7a ed. São Paulo: Martins Fontes; 2007.

14. Silva LF, Cabral IE, Christoffel MM. The (im)possibilities of play for children with outpatient cancer treatment. Acta Paul Enferm [Internet]. 2010 [acesso em 10 out 2013]; 23(3): 33440. Disponível em:

http://www.scielo.br/pdf/ape/v23n3/en_v23n3a04.pdf. mesmas.

15. Minayo MCS. O desafio do conhecimento: pesquisa qualitativa em saúde. $8^{a}$ ed. São Paulo: Hucitec, 2004. 16. Fontanella BJB, Luchesi BM, Saidel MGB, Ricas J, Turato ER, Melo DM. Amostragem em pesquisas qualitativas: proposta de procedimentos para constatar saturação teórica. Cad. Saúde Pública. 2011; 27(2):389-394.

17. Bardin L. Análise de conteúdo. 7a ed. Lisboa: Edições 70; 2010.

18. Nóbrega RD, Collet N, Gomes IP, Holanda ER, Araújo YB.

Criança em idade escolar hospitalizada: significado da condição crônica. Texto Contexto Enferm. [Internet]. 2010 [acesso em 13 out 2013]; 19(3): 425-33. Disponível em http://www.scielo.br/pdf/tce/v19n3/a03v19n3.pdf.

19. Gomes IP, Lima KA, Rodrigues LV, Lima RAG, Collet N. Do diagnóstico à sobrevivência do câncer infantil: perspectiva de crianças. Texto Contexto Enferm. [Internet]. 2013; [acesso em 25 fevereiro 2014];22(3): 671-679. Disponível em: http://www.scielo.br/pdf/tce/v22n3/v22n3a13.pdf. 20. Sousa MLXF, Silva KL, Nóbrega MML, Collet N. Self care deficits in children and adolescents with chronic kidney disease. Text Context Nursing. [Internet]. 2012 [acesso em 14 out 2013]; 21(1):95-102. Disponível em: http://www.indexf.com/textocontexto/2012pdf/e21-095.pdf.

21. Li WH, Chung JO, Ho EK. The effectiveness of therapeutic play, using virtual reality computer games, in promoting the psychological well-being of children hospitalised with cancer. Journal of Clinical Nursing. 2011; 20(15-16):2135-2143.

22. Brito TRP, Resck ZMR, Moreira DS, Marques SM. As práticas lúdicas no cotidiano do cuidar em enfermagem pediátrica. Esc Anna Nery Rev Enferm. 2009; 13(4):802-08.

23. Silva FAC, Andrade PR, Barbosa TR, Hoffmann MV, Macedo CR. Representação do processo de adoecimento de crianças e adolescentes oncológicos junto aos familiares. Esc Anna Nery Rev Enferm. 2009; 13 (2):334-41

24. Paiva RS, Valadares GV, Pontes JS. The need to become family caregivers: qualitative study focusing on the theory based on data. Online braz j nurs. [internet]. 2012 [acesso em 14 out 2013]; 11(3):607-20. Disponível em:

http://www.objnursing.uff.br/index.php/nursing/article/view/363 8.

Artigo recebido em 23/10/2013.

Aprovado para publicação em 07/02/2014.

Artigo publicado em 30/06/2014. 